\title{
SDSS-IV MaNGA: The Intrinsic Shape of Slow Rotator Early-type Galaxies
}

\section{DOI:}

10.3847/2041-8213/aad54b

\section{Document Version}

Final published version

Link to publication record in Manchester Research Explorer

\section{Citation for published version (APA):}

Li, H., Mao, S., Cappellari, M., Graham, M. T., Emsellem, E., \& Long, R. J. (2018). SDSS-IV MaNGA: The Intrinsic Shape of Slow Rotator Early-type Galaxies. Astrophysical Journal Letters, 863(2), L19.

https://doi.org/10.3847/2041-8213/aad54b

\section{Published in:}

Astrophysical Journal Letters

\section{Citing this paper}

Please note that where the full-text provided on Manchester Research Explorer is the Author Accepted Manuscript or Proof version this may differ from the final Published version. If citing, it is advised that you check and use the publisher's definitive version.

\section{General rights}

Copyright and moral rights for the publications made accessible in the Research Explorer are retained by the authors and/or other copyright owners and it is a condition of accessing publications that users recognise and abide by the legal requirements associated with these rights.

\section{Takedown policy}

If you believe that this document breaches copyright please refer to the University of Manchester's Takedown Procedures [http://man.ac.uk/04Y6Bo] or contact uml.scholarlycommunications@manchester.ac.uk providing relevant details, so we can investigate your claim.

\section{OPEN ACCESS}




\title{
SDSS-IV MaNGA: The Intrinsic Shape of Slow Rotator Early-type Galaxies
}

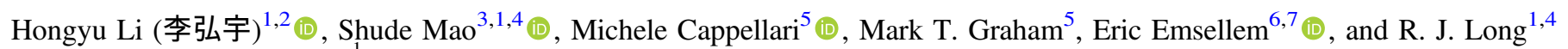 \\ ${ }^{1}$ National Astronomical Observatories, Chinese Academy of Sciences, 20A Datun Road, \\ Chaoyang District, Beijing 100012, People's Republic of China; hyli@nao.cas.cn \\ ${ }^{2}$ University of Chinese Academy of Sciences, Beijing 100049, People's Republic of China \\ ${ }^{3}$ Physics Department and Tsinghua Centre for Astrophysics, Tsinghua University, Beijing 100084, People's Republic of China \\ ${ }_{5}^{4}$ Jodrell Bank Centre for Astrophysics, School of Physics and Astronomy, The University of Manchester, Oxford Road, Manchester M13 9PL, UK \\ ${ }^{5}$ Sub-Department of Astrophysics, Department of Physics, University of Oxford, Denys Wilkinson Building, Keble Road, Oxford, OX1 3RH, UK \\ ${ }^{6}$ Université Lyon 1, Observatoire de Lyon, Centre de Recherche Astrophysique de Lyon and Ecole Normale Supérieure de Lyon, \\ 9 avenue Charles André, F-69230 Saint-Genis Laval, France \\ ${ }^{7}$ European Southern Observatory, Karl-Schwarzschild-Str. 2, D-85748 Garching, Germany \\ Received 2018 February 7; revised 2018 July 14; accepted 2018 July 19; published 2018 August 13
}

\begin{abstract}
By inverting the distributions of galaxies' apparent ellipticities and misalignment angles (measured around the projected half-light radius $R_{\mathrm{e}}$ ) between their photometric and kinematic axes, we study the intrinsic shape distribution of 189 slow rotator early-type galaxies with stellar masses $2 \times 10^{11} M_{\odot}<M_{*}<2 \times 10^{12} M_{\odot}$, extracted from a sample of about 2200 galaxies with integral-field stellar kinematics from the data release 14 (DR14) of the fourth-generation Sloan Digital Sky Survey IV (SDSS-IV) Mapping Nearby Galaxies at Apache Point Observatory (MaNGA) integral field unit (IFU) survey. Thanks to the large sample of slow rotators, Graham et al. showed that there is clear structure in the misalignment angle distribution, with two peaks at both $0^{\circ}$ and $90^{\circ}$ misalignment (characteristic of oblate and prolate rotation, respectively). Here we invert the observed distribution from Graham et al. The large sample allows us to go beyond the known fact that slow rotators are weakly triaxial and to place useful constraints on their intrinsic triaxiality distribution (around $1 R_{\mathrm{e}}$ ) for the first time. The shape inversion is generally non-unique. However, we find that, for a wide set of model assumptions, the observed distribution clearly requires a dominant triaxial-oblate population. For some of our models, the data suggest a minor triaxial-prolate population, but a dominant prolate population is ruled out.
\end{abstract}

Key words: Galaxy: evolution - Galaxy: kinematics and dynamics - Galaxy: structure

\section{Introduction}

The intrinsic shape distribution of early-type galaxies (ETGs) has been studied for decades by statistically inverting the distribution of their ellipticities (e.g., Sandage et al. 1970; Binney \& de Vaucouleurs 1981; Fasano \& Vio 1991; Lambas et al. 1992; Ryden 1992; Kimm \& Yi 2007) or the distribution of their ellipticities and misalignment angles between photometric and kinematic axes (e.g., Weijmans et al. 2014; Foster et al. 2017). Although the recovered intrinsic shape distributions from such methods are generally non-unique (Franx et al. 1991), the fact that all of the fast rotators have kinematic axes aligned with their photometric axes (Cappellari et al. 2007; Emsellem et al. 2007; Krajnović et al. 2011; Fogarty et al. 2015) can only be explained if, as a class, they are nearly axisymmetric (with or without bars). For slow rotators, however, the two axes can be misaligned. Previous studies show that slow rotators are weakly triaxial (Weijmans et al. 2014; Foster et al. 2017), but it is difficult to see any significant features of the misalignment angle distribution due to their limited sample sizes. A more detailed review on this topic can be found in Section 3.3 of Cappellari (2016).

In recent cosmological hydrodynamic simulations (e.g., Figure 7 of Schaller et al. 2015; Figure 10 of Li et al. 2016; Velliscig et al. 2015; and Li et al. 2018a), many massive galaxies are found to be prolate-like, manifesting as mostly slow rotators with different kinematic misalignment angles and formed by major mergers with radial orbits ( $\mathrm{Li}$ et al. 2018a). This theory is broadly consistent with the observation that genuine kinematic misalignment between photometry and kinematics, including some nearly $90^{\circ}$ misalignments
(Krajnović et al. 2011), only happens in slow rotators, which are found above a characteristic stellar mass $\left(M_{\text {crit }} \approx 2 \times\right.$ $10^{11} M_{\odot}$; e.g., Figure 11 of Emsellem et al. 2011; Cappellari et al. 2013a; Cappellari 2016). It would be useful to study the intrinsic shape distribution of slow rotators observationally and put constraints on galaxy formation models.

Some recent works have interpreted the existence of a handful of massive galaxies with $90^{\circ}$ misalignment as circumstantial evidence for the existence of prolate galaxies, e.g., in the Calar Alto Legacy Integral Field Area Survey (CALIFA; Tsatsi et al. 2017) and the MUSE Most Massive Galaxies (M3G) survey (Krajnovic et al. 2018). Especially in the M3G survey, half of the massive $\left(M>10^{12} M_{\odot}\right)$ galaxies have $90^{\circ}$ misalignments. However, a $90^{\circ}$ misalignment is also naturally expected as a significant fraction for triaxial galaxies (see e.g., this Letter). This implies that, unless all slow rotators were $90^{\circ}$ misaligned, which is not the case (see Figure 6 of Cappellari 2016), one cannot interpret $90^{\circ}$ misalignments as evidence for prolate galaxies. Conclusions on galaxy shapes cannot be reached without a statistical study, but the number statistics are so far too limited for this kind of analysis.

MaNGA (Bundy et al. 2015) is currently the largest IFU survey observed by the $2.5 \mathrm{~m}$ Sloan Telescope (Gunn et al. 2006), and as reported by Graham et al. (2018), provides a sufficiently large number of slow rotators to detect actual structure in the distribution of kinematic misalignments. In this Letter we analyze the distributions of ellipticity and misalignment angle measured around the effective radius to provide the first statistical study of the shape distribution of slow rotators, 

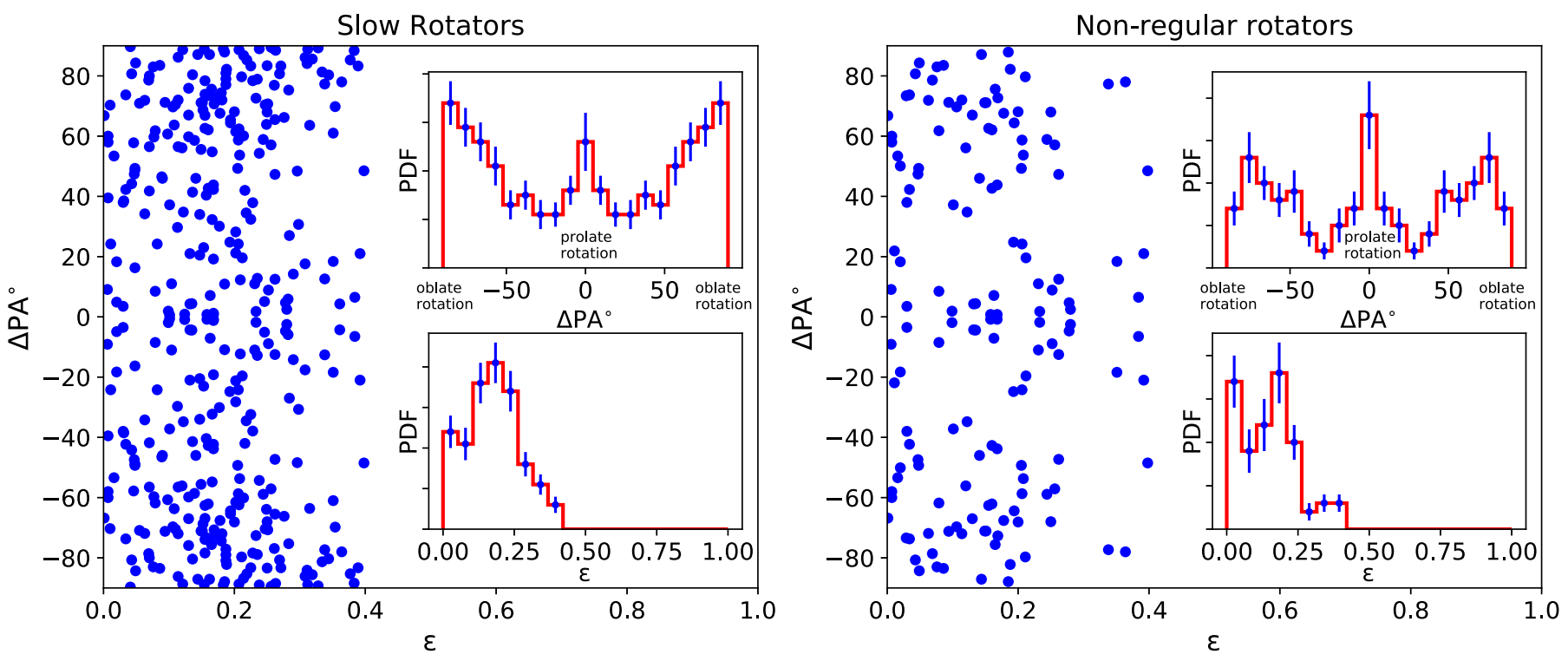

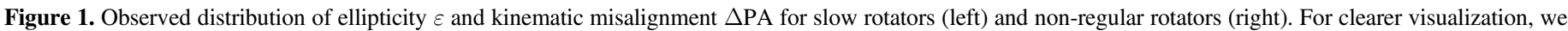

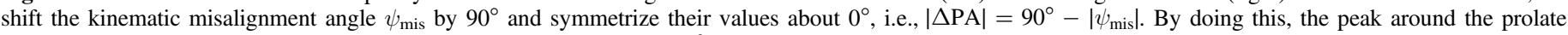

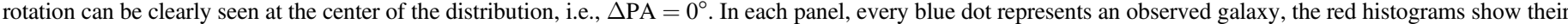
$1 \mathrm{D}$ distribution, and the blue error bars show the error estimated using the bootstrapping method.

which goes beyond the well-established fact that they are "weakly triaxial" (e.g., review by Cappellari 2016).

The structure of this Letter is as follows. In Section 2, we introduce the galaxy sample and the methods we use. In Section 3, we show the results. In Section 4, we summarize our results and draw conclusions.

\section{Sample and Methods}

\subsection{Sample Selection and Data}

We use the galaxy sample from Graham et al. (2018), based on SDSS-IV DR14 MaNGA (Abolfathi et al. 2018), which has $\sim 2700$ galaxies. The measured ellipticity $\varepsilon$, misalignment angle $\Psi_{\text {mis }}$ (around the effective radius), beam-corrected angular momentum parameter $\lambda_{\mathrm{R}_{e}}$, and kinematic classification are from Table 2 of Graham et al. (2018). We select massive slow rotators using two alternative techniques. The first is quantitative: we select galaxies with $\lambda_{\mathrm{R}_{e}}<0.08+\varepsilon / 4$ and $\varepsilon<0.4$ (Cappellari 2016), as indicated by the black solid lines in Figure 11 of Graham et al. (2018). The second one is qualitative: we select galaxies with visually classified ETGs as non-regular rotators. These galaxies do not exhibit a spider diagram velocity map, and this is thought to be an indicator of a slow rotator (see Graham et al. 2018 and Figure 4 of Cappellari 2016 for more details). In both samples, we remove galaxies with $M \equiv 2 \times M_{1 / 2}<2 \times 10^{11} M_{\odot}$, where $M_{1 / 2}$ is the dynamical mass within the 3D half-light radius $\left(r_{1 / 2}\right)$ from Table 1 of Li et al. (2018b). When the dark matter is small within the effective radius, $M$ defined above is a good proxy of the stellar mass (Cappellari et al. 2013b). This cut on mass is needed because most slow or non-regular rotators with $M<2 \times 10^{11} M_{\odot}$ are not genuine slow rotators (Graham et al. 2018). Finally, we have 189 galaxies in the slow rotator sample and 74 galaxies in the non-regular sample. We note here that 72 of the 74 non-regular rotators are also in the 189 slow rotator sample.

In Figure 1, we show the 1D histograms and the 2D distribution of the observed ellipticity $\varepsilon$ and kinematic misalignment $\psi_{\text {mis }}$ for slow rotators and non-regular rotators from Graham et al. (2018). We shift and symmetrize the misalignment angle distribution. We note that we did not modify the data values in the symmetrization, but just plot the data twice between $\left[-90^{\circ}, 0^{\circ}\right]$ and $\left[0^{\circ}, 90^{\circ}\right]$ for clearer visualization. In the model fitting, we still use the original distribution as input. For normal axisymmetric rotation, $\Delta \mathrm{PA}= \pm 90^{\circ}$. The uncertainties estimated by the bootstrapping method are shown by the blue error bars. As one can see, the peak around $\triangle \mathrm{PA}=0^{\circ}$ has a confidence level more than twosigma.

\subsection{Projection of Luminosity Density and Velocity Field}

The projection of the luminosity density of a triaxial galaxy has been discussed in Stark (1977), Binney (1985), and Franx (1988). Here we just make a brief summary. Following Binney (1985), we assume the luminosity density of a triaxial galaxy could be described by similar coaxial ellipsoids, i.e., $\rho=\rho\left(\mathrm{m}^{2}\right)$, where $m^{2}=x^{2} / a^{2}+y^{2} / b^{2}+z^{2} / c^{2}$, and $x$-, $y$-, and $z$-axes are aligned with the principal axes with $a>b>c$. The axis ratios are defined as

$$
\zeta=b / a, \quad \xi=c / a, \quad \eta=c / b .
$$

When a galaxy is viewed along the direction $(\theta, \phi)$ in polar coordinates, the projected ellipticity $\varepsilon$ and the minor axes position angle $\Gamma_{\text {minor }}$ with respect to the projected $z$-axis are completely determined by the intrinsic axes ratios (Equations (12) and (13) in Binney 1985)

$$
\begin{gathered}
\varepsilon(\theta, \phi ; \xi, \zeta)=1-\sqrt{\frac{A+C-\sqrt{(A-C)^{2}+B^{2}}}{A+C+\sqrt{(A-C)^{2}+B^{2}}}}, \\
\Gamma_{\text {minor }}(\theta, \phi ; \xi, \zeta)=\frac{1}{2} \arctan \left(\frac{B}{A-C}\right),
\end{gathered}
$$



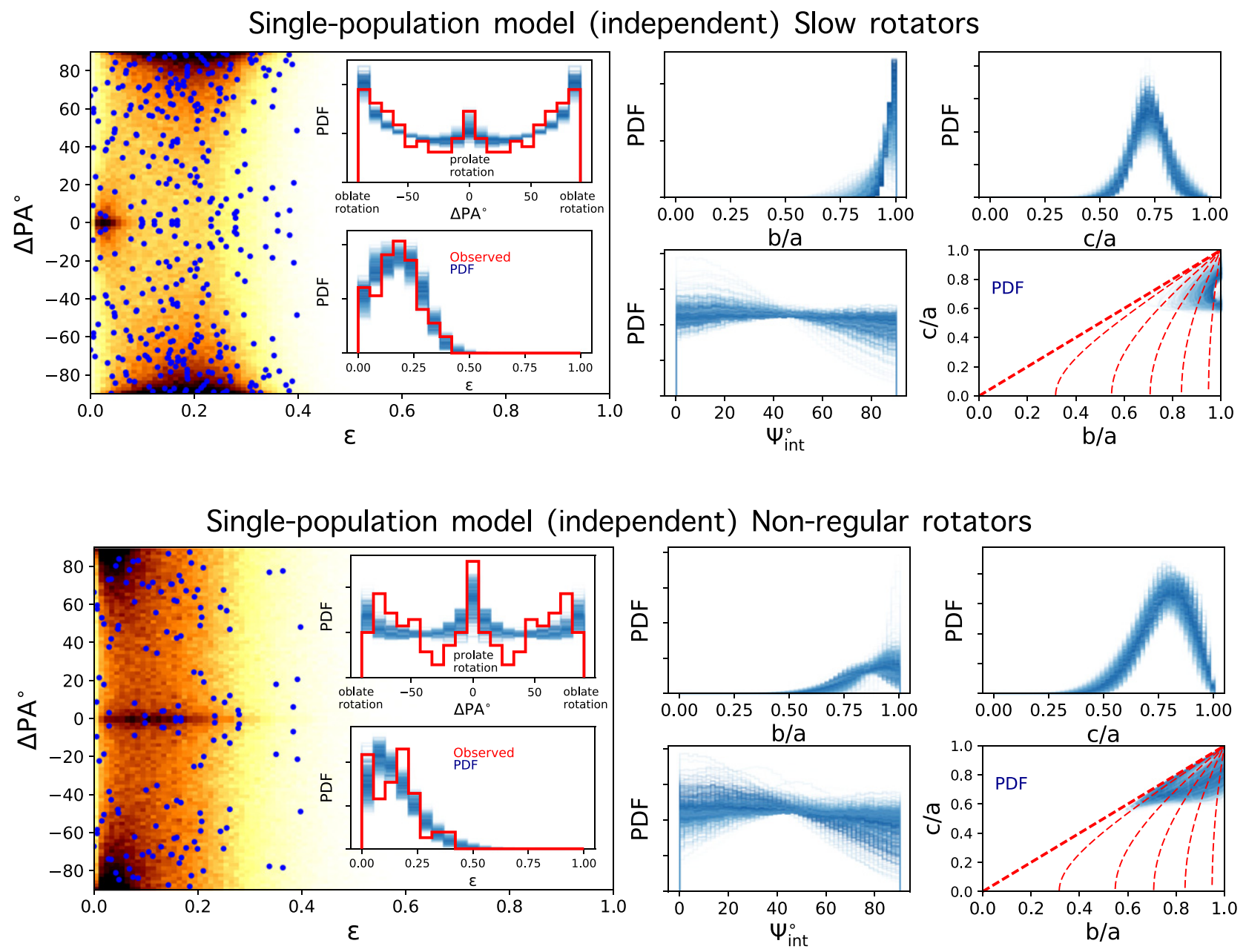

Figure 2. Single-population models with independent intrinsic misalignment for massive slow rotators classified using two alternative techniques, namely either (i) from the $\left(\lambda_{\mathrm{R}_{e}}, \varepsilon\right)$ diagram (top) or (ii) visually classified from their kinematic maps (bottom). In each left panel, the blue dots show the observed distribution, and the color map shows the model distribution that maximizes the likelihood. The red histograms show the observed distribution. The blue lines show the probability distribution, which are randomly selected from the MCMC chain and color coded by their likelihood. The bluer the color, the larger the likelihood. We use the same method described in Figure 1 to shift and symmetrize the distribution for visualization purposes only. We note that we still use the original distribution in the modelfitting process. The predicted axis ratio and intrinsic misalignment are shown by the blue lines in the right panels. In the $b / a$ vs. $c / a$ panel (lower right), every blue line shows the $1 \sigma(68 \%)$ contour of the $2 \mathrm{D}$ axis ratio distribution of that model. The red thin-dashed lines, from left to right, show the line of constant triaxial parameter $T=\frac{a^{2}-b^{2}}{a^{2}-c^{2}}: 0.1,0.3,0.5,0.7$, and 0.9 . The red thick-dashed line shows the position where the axis ratio $b / a=c / a$.

with

$$
\begin{gathered}
A \equiv \frac{\cos ^{2} \theta}{\xi^{2}}\left(\sin ^{2} \phi+\frac{\cos ^{2} \phi}{\zeta^{2}}\right)+\frac{\sin ^{2} \theta}{\zeta^{2}}, \\
B \equiv \cos \theta \sin 2 \phi\left(1-\frac{1}{\zeta^{2}}\right) \frac{1}{\xi^{2}} \\
C \equiv\left(\frac{\sin ^{2} \phi}{\zeta^{2}}+\cos ^{2} \phi\right) \frac{1}{\xi^{2}}
\end{gathered}
$$

In a triaxial galaxy, the angular momentum vector is not required to be aligned with the principal axes, but can lie anywhere in the $x-z$ plane (Statler 1987). Following Franx et al. (1991), we define $0^{\circ} \leqslant \psi_{\text {int }} \leqslant 90^{\circ}$ as the angle between the $z$-axis and the angular momentum vector. The position angle of the apparent angular momentum $\Gamma_{\text {kin }}$, with respect to the projected $z$-axis, only depends on the viewing angle and the intrinsic misalignment (Equation (6) in Franx et al. 1991)

$$
\tan \Gamma_{\mathrm{kin}}=\frac{\sin \phi \sin \psi_{\mathrm{int}}}{-\cos \phi \cos \theta \sin \psi_{\mathrm{int}}+\sin \theta \cos \psi_{\mathrm{int}}} .
$$

The kinematic misalignment angle $\Psi$ between the projected minor axis and the projected angular momentum is given by

$$
\sin \Psi=\left|\sin \left(\Gamma_{\text {kin }}-\Gamma_{\text {minor }}\right)\right|, \quad 0^{\circ} \leqslant \Psi \leqslant 90^{\circ},
$$

where $\Psi$ corresponds to our observed quantity $\left|\psi_{\text {mis }}\right|$ from Graham et al. (2018).

\subsection{Monte Carlo Simulations}

From Section 2.2, we know the apparent kinematic misalignment angle $\Psi$ and apparent ellipticity $\varepsilon$ are determined by the intrinsic axis ratios $(\zeta, \xi)$, intrinsic misalignment angle of the angular momentum $\psi_{\text {int }}$ and viewing angle $(\theta, \phi)$, i.e., $\Psi=\Psi\left(\zeta, \xi, \psi_{\text {int }}, \theta, \phi\right), \varepsilon=\varepsilon(\zeta, \xi, \theta, \phi)$. If we assume that the viewing angle is random (i.e., flat distributions in $[-1,1]$ for $\cos \theta$ and $[0,2 \pi]$ for $\phi)$, and the distribution of the axis ratios and intrinsic misalignment of the galaxies in our sample can be 
Two-population model (independent) Slow rotators
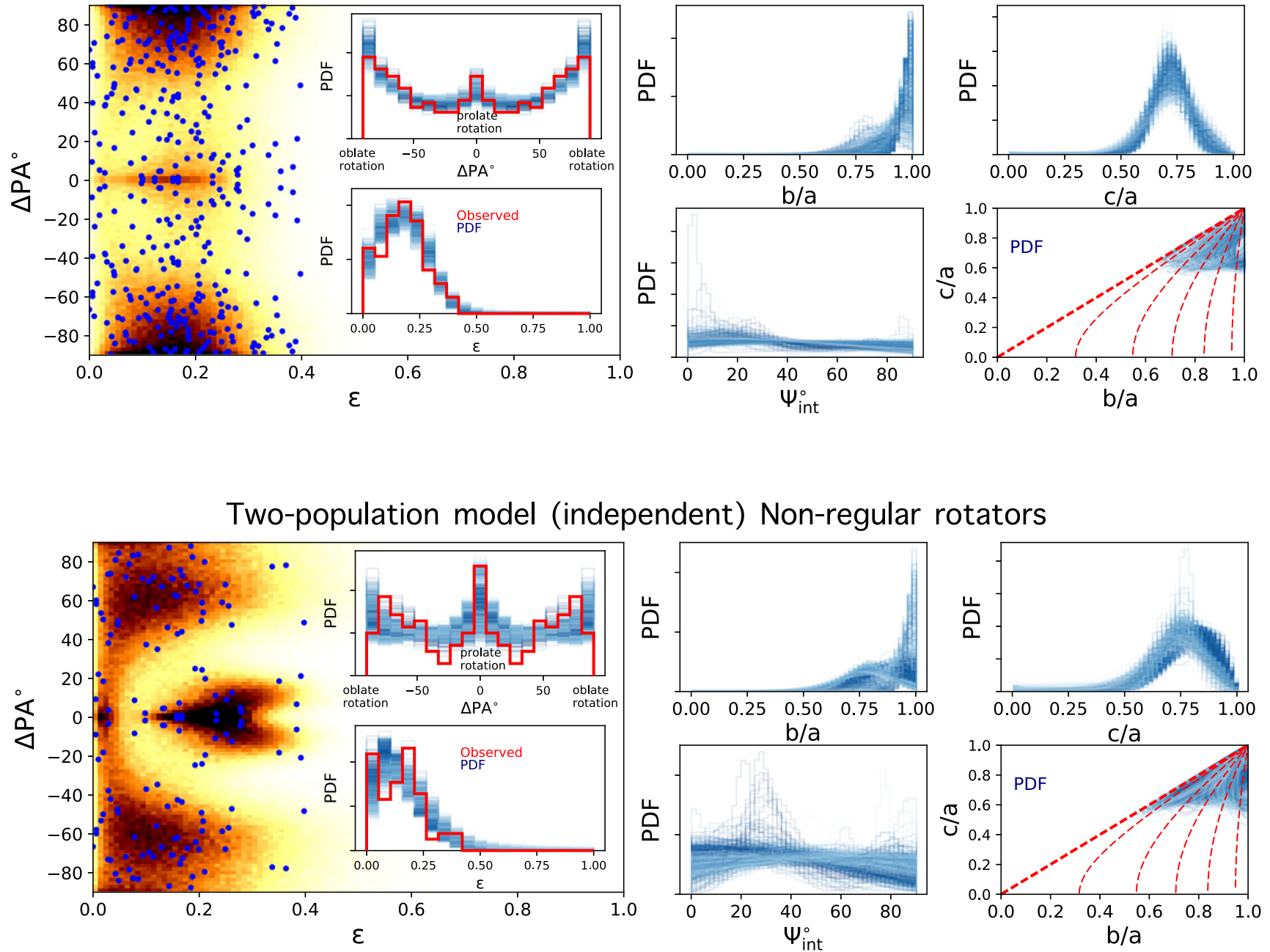

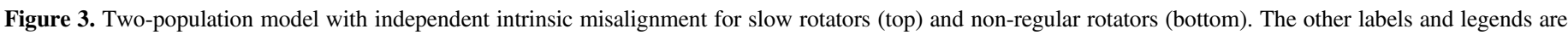
the same as Figure 2.

described by some distribution functions, e.g., $p(\zeta), p(\xi)=$ $p(\xi / \zeta \mid \zeta) p(\zeta)=p(\eta \mid \zeta) p(\zeta)$ and $p\left(\psi_{\text {int }}\right)$, we can calculate the model probability distribution of $\Psi$ and $\varepsilon$

$$
p(\Psi, \varepsilon)=p\left[\Psi, \varepsilon \mid p(\zeta), p(\eta), p\left(\psi_{\text {int }}\right)\right] .
$$

The reason for using $p(\eta)$ instead of $p(\xi)$ is because $\eta$ is between 0 and 1 and is independent of $\zeta$, which makes it easier and faster to implement numerically, while $\xi$ has to be smaller than $\zeta$. We also confirmed by visual examination that the distribution $p(\xi)$ derived from $p(\zeta)$ and $p(\eta)$ in our model is physically reasonable and can cover various shapes of distributions.

We use two different models to parameterize $p(\zeta), p(\eta)$ and $p\left(\psi_{\text {int }}\right)$. The first one is a single-population model, in which we assume $p(\zeta), p(\eta)$ and $p\left(\psi_{\text {int }}\right)$ are independent and can be described by single truncated Gaussian functions

$$
p\left(\zeta \mid \mu_{\zeta}, \sigma_{\zeta}\right) \propto \frac{1}{\sqrt{2 \pi \sigma_{\zeta}^{2}}} \exp \left(\frac{\left(\zeta-\mu_{\zeta}\right)^{2}}{\sigma_{\zeta}^{2}}\right), \quad 0 \leqslant \zeta \leqslant 1
$$

$$
\begin{aligned}
& p\left(\eta \mid \mu_{\eta}, \sigma_{\eta}\right) \propto \frac{1}{\sqrt{2 \pi \sigma_{\eta}^{2}}} \exp \left(\frac{\left(\eta-\mu_{\eta}\right)^{2}}{\sigma_{\eta}^{2}}\right), \quad 0 \leqslant \eta \leqslant 1 \\
& p\left(\psi_{\text {int }} \mid \mu_{\left.\psi_{\text {int }}, \sigma_{\psi_{\text {int }}}\right)}\right. \\
& \propto \frac{1}{\sqrt{2 \pi \sigma_{\psi_{\text {int }}}^{2}}} \exp \left(\frac{\left(\psi_{\text {int }}-\mu_{\psi_{\text {int }}}\right)^{2}}{\sigma_{\psi_{\text {int }}}^{2}}\right), \quad 0 \leqslant \psi_{\text {int }} \leqslant \frac{\pi}{2} .
\end{aligned}
$$

The probability outside of the boundary is truncated and set to 0 . There are six free parameters for a single-population model, they are $\mu_{\zeta}, \sigma_{\zeta}, \mu_{\eta}, \sigma_{\eta}, \mu_{\psi_{\text {int }}}$ and $\sigma_{\psi_{\text {int }}}$.

The second model is a two-population model, in which we assume that $p(\zeta), p(\eta)$, and $p\left(\psi_{\text {int }}\right)$ are independent and can be described by two truncated Gaussian functions, with each Gaussian representing a galaxy population (e.g., oblate, triaxial, or prolate)

$$
\begin{aligned}
& p\left(\zeta \mid \mu_{\zeta_{1}}, \sigma_{\zeta_{1}}, \mu_{\zeta_{2}}, \sigma_{\zeta_{2}}\right) \\
& \quad=f_{1} p\left(\zeta \mid \mu_{\zeta_{1}}, \sigma_{\zeta_{1}}\right)+\left(1-f_{1}\right) p\left(\zeta \mid \mu_{\zeta_{2}}, \sigma_{\zeta_{2}}\right),
\end{aligned}
$$


Single-population model (Weijmans+14) Slow rotators
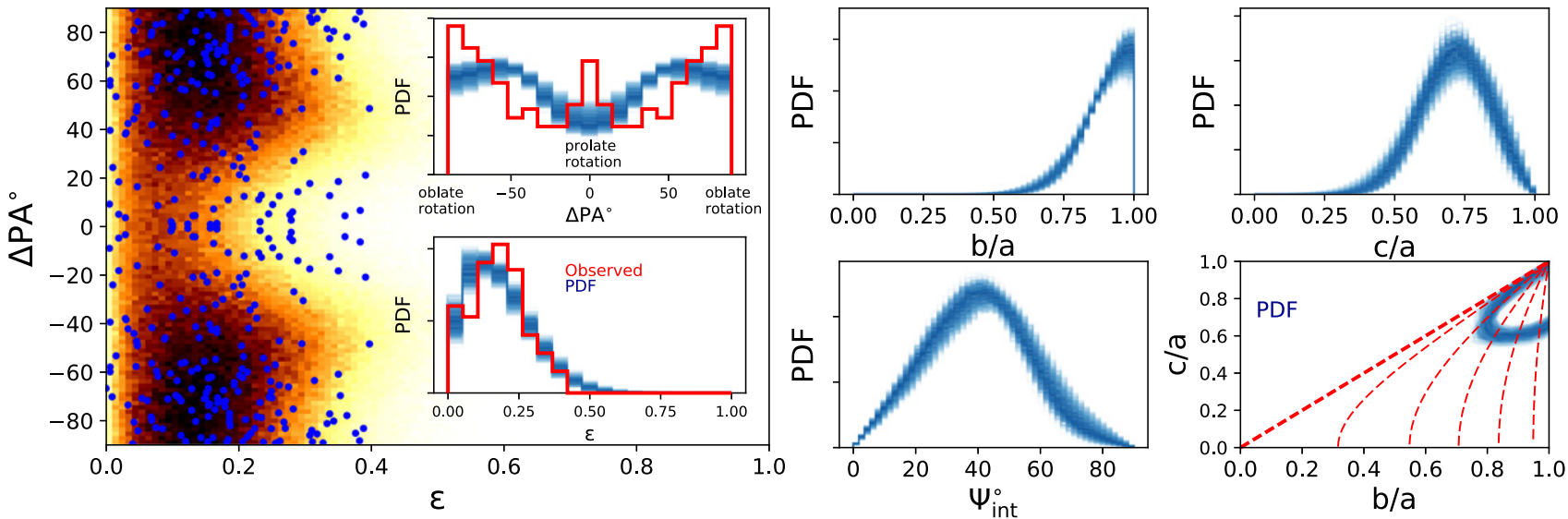

Two-population model (Weijmans+14) Slow rotators
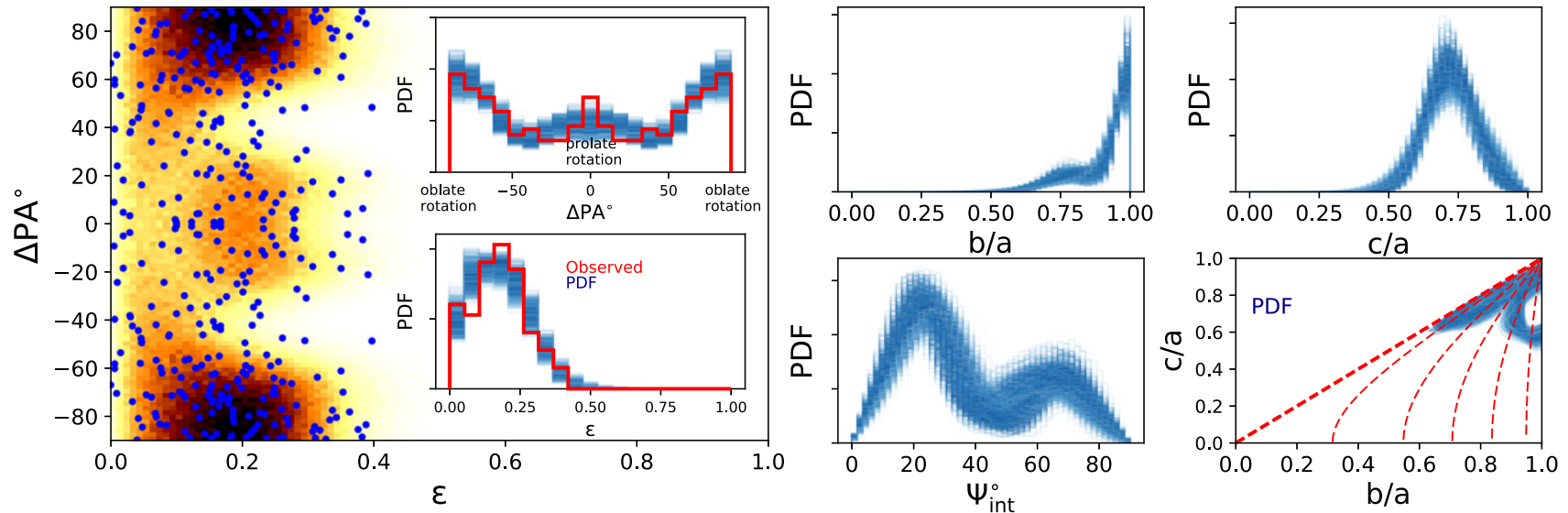

Figure 4. Single-population (top) and two-population (bottom) models with the Weijmans et al. (2014) intrinsic misalignment for slow rotators. The other labels and legends are the same as Figure 2.

Table 1

Best-fit (Maximum-likelihood) Parameters for the Models Shown in Figures 2-4

\begin{tabular}{|c|c|c|c|c|c|c|c|}
\hline Model & $\mu_{\zeta}$ & $\sigma_{\zeta}$ & $\mu_{\eta}$ & $\sigma_{\eta}$ & $\mu_{\psi_{\text {int }}}$ & $\sigma_{\psi_{\text {int }}}$ & $f_{1}$ \\
\hline SR SINGLE-INDEPENDENT & $0.99_{-0.02}^{+0.01}$ & $0.03_{-0.01}^{+0.03}$ & $0.74_{-0.01}^{+0.02}$ & $0.08_{-0.01}^{+0.01}$ & $0.14_{-0.40}^{+0.58}$ & $4.66_{-1.26}^{+1.13}$ & $\cdots$ \\
\hline NR SINGLE-INDEPENDENT & $0.83_{-0.06}^{+0.04}$ & $0.13_{-0.03}^{+0.03}$ & $0.99_{-0.04}^{+0.03}$ & $0.04_{-0.03}^{+0.04}$ & $0.07_{-0.44}^{+0.57}$ & $0.87_{-1.35}^{+1.28}$ & $\ldots$ \\
\hline SR TWO1_-INDEPENDENT & $0.99_{-0.24}^{+0.05}$ & $0.04_{-0.06}^{+0.49}$ & $0.78_{-0.07}^{+0.12}$ & $0.10_{-0.03}^{+0.52}$ & $0.35_{-0.32}^{+0.63}$ & $0.24_{-0.64}^{+0.32}$ & $0.55_{-0.42}^{+0.45}$ \\
\hline SR TWO²-INDEPENDENT & $0.86_{-0.20}^{+0.06}$ & $0.08_{-0.06}^{+0.45}$ & $0.82_{-0.06}^{+0.14}$ & $0.04_{-0.03}^{+0.49}$ & $1.55_{-0.39}^{+0.74}$ & $0.92_{-0.57}^{+0.35}$ & $\ldots$ \\
\hline NR TWO ${ }^{1}$-INDEPENDENT & $0.99_{-0.21}^{+0.08}$ & $0.04_{-0.06}^{+0.49}$ & $0.88_{-0.25}^{+0.06}$ & $0.13_{-0.07}^{+0.50}$ & $0.48_{-0.41}^{+0.59}$ & $0.10_{-0.52}^{+0.39}$ & $0.58_{-0.13}^{+0.63}$ \\
\hline NR TWO $^{2}$-INDEPENDENT & $0.78_{-0.19}^{+0.07}$ & $0.09_{-0.05}^{+0.40}$ & $0.91_{-0.21}^{+0.05}$ & $0.05_{-0.06}^{+0.51}$ & $1.22_{-0.38}^{+0.60}$ & $1.09_{-0.45}^{+0.37}$ & $\ldots$ \\
\hline SR SINGLE-W14 & $0.99_{-0.01}^{+0.01}$ & $0.13_{-0.01}^{+0.01}$ & $0.82_{-0.02}^{+0.03}$ & $0.12_{-0.02}^{+0.03}$ & $\cdots$ & $\cdots$ & .. \\
\hline SR TWO-W14 ${ }^{1}$ & $0.99_{-0.19}^{+0.05}$ & $0.05_{-0.06}^{+0.27}$ & $0.75_{-0.10}^{+0.15}$ & $0.08_{-0.02}^{+0.03}$ & $\ldots$ & $\ldots$ & $0.70_{-0.47}^{+0.44}$ \\
\hline SR TWO-W14 ${ }^{2}$ & $0.78_{-0.18}^{+0.02}$ & $0.08_{-0.07}^{+0.09}$ & $0.96_{-0.08}^{+0.15}$ & $0.05_{-0.04}^{+0.09}$ & $\cdots$ & $\ldots$ & $\ldots$ \\
\hline
\end{tabular}

Note. The uncertainties are calculated as the differences between the 16th and 50th, 50th and 84th percentiles $(1 \sigma)$ of the 1D distribution of each parameter, respectively. We note that the best-fit parameters can deviate from the 50th percentile (i.e., the median), as the posterior distributions are irregular. SR and NR represent slow rotators and non-regular rotators, respectively. SINGLE-INDEPENDENT represents the single-population model with independent intrinsic misalignment. $\mathrm{TWO}^{1}$-INDEPENDENT and $\mathrm{TWO}^{2}$-INDEPENDENT represent the two populations in the two-population model with independent intrinsic misalignment. SINGLE-W14, TWO-W14 ${ }^{1}$, and TWO-W14 ${ }^{2}$ represent the single-population model and the two components of the two-population model with the Weijmans et al. (2014) intrinsic misalignment. $\mu_{\psi_{\text {int }}}$ and $\sigma_{\psi_{\text {int }}}$ are in radians. 


$$
\begin{gathered}
p\left(\eta \mid \mu_{\eta_{1}}, \sigma_{\eta_{1}}, \mu_{\eta_{2}}, \sigma_{\eta_{2}}\right) \\
=f_{1} p\left(\eta \mid \mu_{\eta_{1}}, \sigma_{\eta_{1}}\right)+\left(1-f_{1}\right) p\left(\eta \mid \mu_{\eta_{2}}, \sigma_{\eta_{2}}\right) \\
p\left(\psi_{\text {int }} \mid \mu_{\psi_{\text {int } 1}}, \sigma_{\psi_{\text {int }}}, \mu_{\psi_{\text {int } 2}}, \sigma_{\psi_{\text {int }}}\right) \\
=f_{1} p\left(\psi_{\text {int }} \mid \mu_{\psi_{\text {int } 1}}, \sigma_{\psi_{\text {int } 1}}\right)+\left(1-f_{1}\right) p\left(\psi_{\text {int }} \mid \mu_{\psi_{\text {int } 2}}, \sigma_{\psi_{\text {int }}}\right) .
\end{gathered}
$$

where $f_{1}$ represents the fraction of the galaxies in the first galaxy population. The functional form and truncation boundary for each population are exactly the same as in Equations (10)-(12). There are 13 free parameters in this model; they are $\mu_{\zeta_{1}}, \sigma_{\zeta_{1}}, \mu_{\eta_{1}}, \sigma_{\eta_{1}}, \mu_{\psi_{\text {int } 1}}, \sigma_{\psi_{\text {int } 1}}, \mu_{\zeta_{2}}, \sigma_{\zeta_{2}}, \mu_{\eta_{2}}, \sigma_{\eta_{2}}$,

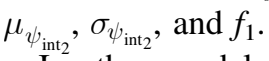

In the models above, we assume that the intrinsic misalignment is independent of the intrinsic shape. However, a one-to-one relation is assumed in previous studies (Weijmans et al. 2014; Foster et al. 2017)

$$
\tan \psi_{\text {int }}=\sqrt{\frac{T}{1-T}},
$$

where $T=\frac{a^{2}-b^{2}}{a^{2}-c^{2}}$ is the triaxial parameter (i.e., the intrinsic shape). We also adapt this one-to-one relation in our singleand two-population models in our Monte Carlo simulations. In summary, the models we use are

1. a single-population model with independent intrinsic misalignment, 6 free parameters;

2. a two-population model with independent intrinsic misalignment, 13 free parameters;

3. a single-population model with intrinsic misalignment following the model of Weijmans et al. (2014), 4 free parameters;

4. a two-population model with intrinsic misalignment following the model of Weijmans et al. (2014), 9 free parameters.

For all of the models, we estimate $p(\Psi, \varepsilon)$ in Equation (9) numerically. With a given set of model parameters $\boldsymbol{p}$, we first sample $n$ points of $\cos \theta, \phi$ from a flat distribution, and $\zeta, \eta$, and $\psi_{\text {int }}$ from $p(\zeta \mid \boldsymbol{p}), p(\eta \mid \boldsymbol{p})$ and $p\left(\psi_{\text {int }} \mid \boldsymbol{p}\right)$, respectively (for the Weijmans et al. (2014) models, $\psi_{\text {int }}$ is calculated directly using Equation (16)). We then calculate the model predicted $\varepsilon$ and $\Psi$ for every point using Equations (2) and (8), respectively, and bin all of the points on a 2D histogram ( 25 by 25 ). Finally, we normalize the histogram, and use bilinear interpolation to calculate the probability for a given $(\Psi, \varepsilon)$. We set $n=9,000,000$ in our calculation, which is enough to produce a smooth probability distribution.

We perform the inversion, i.e., infer $p(\zeta), p(\xi)$ and $p\left(\psi_{\text {int }}\right)$ from the observed distribution, by maximizing the likelihood of the observed distribution, defined as

$$
\ln L=\sum_{i} \ln p\left(\left|\psi_{\mathrm{mis}}^{i}\right|, \varepsilon_{i}\right)
$$

where $\psi_{\text {mis }}^{i}$ and $\varepsilon_{i}$ are the apparent kinematic misalignment angle and ellipticity for the $i$ th observed galaxy. $p(\Psi, \varepsilon)$ is defined in Equation (9) and estimated numerically using the method described above. The sum is over all the galaxies in the sample (i.e., slow rotators or non-regular rotators described in Section 2.1). We use a python implementation of the Markov chain Monte Carlo (MCMC) algorithm (emcee; ForemanMackey et al. 2013) to obtain the parameters that maximize the likelihood as well as the probability distribution of the model parameters. We use 200 walkers and run for 3000 and 12,000 steps for the single-population model and two-population model, respectively. In addition to emcee, we also tried another MCMC package AdaMet (Cappellari et al. 2013b). Those two packages give similar results. The parameters are sampled within their mathematical limits. For the single-population model, they are: $[0,1]$ for $\mu_{\zeta}$ and $\mu_{\eta} ;[0,1]$ for $\sigma_{\zeta}$ and $\sigma_{\eta}$; $[0, \pi / 2]$ for $\mu_{\psi_{\mathrm{int}}} ;\left[0, \frac{3}{2} \pi\right]$ for $\sigma_{\psi_{\mathrm{int}}}$. For the two-population model, the boundaries are $[0,1]$ for $\mu_{\zeta_{1}}, \mu_{\eta_{1}}, \mu_{\zeta_{2}}$ and $\mu_{\eta_{2}} ;[0,1]$ for $\sigma_{\zeta_{1}}, \sigma_{\eta_{1}}, \sigma_{\zeta_{2}}$ and $\sigma_{\eta_{2}} ;[0, \pi / 2]$ for $\mu_{\psi_{\text {int }_{1}}}$ and $\mu_{\psi_{\text {int } 2}} ;[0, \pi / 2]$ for $\sigma_{\psi_{\text {int }_{1}}}$ and $\sigma_{\psi_{\text {int }}} ;[0,1]$ for $f_{1}$.

\section{Results}

In this section, we show the fitting results of the observed distribution for all the models. To test the robustness of our results, we fit both the slow rotator samples selected with different techniques in Section 2.1. The fitting is performed using the methods described in Section 2.3.

The model that maximizes the likelihood and the probability distribution function from MCMC for the single-population model with independent intrinsic misalignment is shown in Figure 2. From the probability distribution (blue histograms) in the left panels, the model can produce three peaks (around $\triangle \mathrm{PA}=0^{\circ}, \pm 90^{\circ}$ ), which qualitatively agree with the observed data. However, the model fits the detailed structures less well, e.g., the dip between $\sim 20^{\circ}$ and $\sim 40^{\circ}$, especially for nonregular rotators. Furthermore, in the right panels, one can see that the model predicted axis ratios from different samples do not agree with each other: galaxies in the slow rotator sample prefer a triaxial-oblate shape with a flat intrinsic misalignment distribution, while galaxies in the non-regular sample prefer a triaxial-prolate shape with flat intrinsic misalignment.

Similar diagrams for the two-population model with independent intrinsic misalignment are shown in Figure 3. As can be seen from the probability distribution (blue histogram) in the left panels, the model can not only give three peaks, but also match the amplitude of the peaks and other detailed structures relatively well. We also calculate the $p$-value from the KS statistic for the $1 \mathrm{D}$ distribution of $\triangle \mathrm{PA}$. We find that the $p$-value increases from $\sim 0.8$ to $\sim 1$ after we change to the twopopulation model. From the probability distribution of the axis ratio distributions (blue histogram and contours) in the right panels, the galaxy sample is dominated by a triaxial-oblate population, while there are also some minor galaxy populations located in other regions. But due to large model degeneracy, it is difficult to constrain their axis ratios well. We note that in practice it is difficult for the MCMC chain to converge under such large model degeneracy, unless it is run with extremely long steps. In general, the probability distributions of intrinsic axis ratios are similar for slow rotators and non-regular rotators, despite the different selection criteria.

In Figure 4, we show the results of single- and twopopulation models with Weijmans et al. (2014) intrinsic 
misalignment for the slow rotator sample. Unlike the independent intrinsic misalignment model in Figure 2, the single-population model with Weijmans et al. (2014) intrinsic misalignment fails to reproduce the observed peaks. In order to explain the observations, the model requires $\sim 70 \%$ of the galaxies in the sample have triaxial-oblate shape, while the other $\sim 30 \%$ have triaxial-prolate shape. Although model dependent, this gives us a hint that there might be a minor population in the slow rotators that has triaxial-prolate shape. The best-fit model parameters and their uncertainties from MCMC are listed in Table 1.

In order to test whether our results are affected by outliers (e.g., galaxies with no rotation or poor data qualities), we rerun our model including an outlier component with constant likelihood. The results are still the same and the outlier fraction (free parameter in the fitting) is less than $5 \%$ for all the models and samples. We also try a different axis ratio parametrization described in Lambas et al. (1992). The conclusions remain unchanged.

\section{Conclusions}

We study the distribution of apparent ellipticity and kinematic misalignment for slow rotators measured in Graham et al. (2018), based on a SDSS-IV DR14 MaNGA sample. The distribution shows clear structure in the misalignment angle distribution: there are two peaks at both $0^{\circ}$ and $90^{\circ}$ misalignment (characteristic of oblate and prolate rotation, respectively). By assuming Gaussian shapes of the axis ratios and the intrinsic misalignment distribution, we invert the observed distribution to obtain the intrinsic one using Monte Carlo simulations.

We find that our models with different assumptions clearly require a dominant triaxial-oblate population, beyond the known fact that slow rotators are weakly triaxial. If we assume that the intrinsic shape and the intrinsic misalignment are independent, the constraints on the intrinsic axis ratios are less strong. The observed distribution can be explained by a dominant triaxial-oblate population (with some minor populations of different shapes). However, if a one-to-one intrinsic shape-misalignment relation is assumed, the model requires two distinct galaxy populations (i.e., a dominant triaxial-oblate population and a minor triaxial-prolate population) in order to explain the observations, suggesting a hint for a minor triaxialprolate population.

The uncertainties in our study mainly come from the observational uncertainties of the misalignment angle and the ellipticity plus the intrinsic degeneracies in the inversion problem. In the study, we measure the ellipticity, photometric, and kinematic position angles around the effective radius, and these values could vary with radius and cause uncertainties in the analysis. In addition, galaxies with a round shape and very slow rotation have large uncertainties in the misalignment angle measurement. These uncertainties are difficult to estimate robustly and incorporate in the model. Better observations and larger samples may help to reduce these uncertainties as well as the Poisson noise shown in Figure 1. The model degeneracies are intrinsic and difficult to remove. Our simulations show that the fitting results depend on the intrinsic misalignment model. This suggests a better understanding of the intrinsic shapemisalignment relationship can be useful in the inversion. A study in numerical simulation may be helpful. The MaNGA sample we used is not volume limited, but has a flat stellar mass distribution (Bundy et al. 2015). Because the galaxies we selected have a narrow mass range (between $2 \times 10^{11} M_{\odot}$ and $\sim 10^{12} M_{\odot}$ ), this would only have a minor effect on the conclusions.

This work was supported by the National Science Foundation of China (grant No. 11333003, 11390372 to S.M.). M.C. acknowledges support from a Royal Society University Research Fellowship. We performed our computer runs on the Zen high-performance computer cluster of the National Astronomical Observatories, Chinese Academy of Sciences (NAOC), and the Venus server at Tsinghua University. This research made use of Marvin (Cherinka et al. 2018), a core Python package and web framework for MaNGA data, developed by Brian Cherinka, José Sánchez-Gallego, and Brett Andrews.

Funding for the Sloan Digital Sky Survey IV has been provided by the Alfred P. Sloan Foundation, the U.S. Department of Energy Office of Science, and the Participating Institutions. SDSS-IV acknowledges support and resources from the Center for High-Performance Computing at the University of Utah. The SDSS web site is http://www. sdss.org.

SDSS-IV is managed by the Astrophysical Research Consortium for the Participating Institutions of the SDSS Collaboration including the Brazilian Participation Group, the Carnegie Institution for Science, Carnegie Mellon University, the Chilean Participation Group, the French Participation Group, Harvard-Smithsonian Center for Astrophysics, Instituto de Astrofísica de Canarias, The Johns Hopkins University, Kavli Institute for the Physics and Mathematics of the Universe (IPMU)/University of Tokyo, Lawrence Berkeley National Laboratory, Leibniz Institut für Astrophysik Potsdam (AIP), Max-Planck-Institut für Astronomie (MPIA Heidelberg), Max-Planck-Institut für Astrophysik (MPA Garching), MaxPlanck-Institut für Extraterrestrische Physik (MPE), National Astronomical Observatories of China, New Mexico State University, New York University, University of Notre Dame, Observatário Nacional/MCTI, The Ohio State University, Pennsylvania State University, Shanghai Astronomical Observatory, United Kingdom Participation Group, Universidad Nacional Autónoma de México, University of Arizona, University of Colorado Boulder, University of Oxford, University of Portsmouth, University of Utah, University of Virginia, University of Washington, University of Wisconsin, Vanderbilt University, and Yale University.

\section{ORCID iDs}

Hongyu Li (李弘宇) (10 https://orcid.org/0000-0002-65189866

Shude Mao (iD https://orcid.org/0000-0001-8317-2788

Michele Cappellari (i) https://orcid.org/0000-0002-1283-8420

Eric Emsellem (1) https://orcid.org/0000-0002-6155-7166

\section{References}

Abolfathi, B., Aguado, D. S., Aguilar, G., et al. 2018, ApJS, 235, 24 Binney, J. 1985, MNRAS, 212, 767

Binney, J., \& de Vaucouleurs, G. 1981, MNRAS, 194, 679

Bundy, K., Bershady, M. A., Law, D. R., et al. 2015, ApJ, 798, 7

Cappellari, M. 2016, ARA\&A, 54, 597

Cappellari, M., Emsellem, E., Bacon, R., et al. 2007, MNRAS, 379, 418

Cappellari, M., McDermid, R. M., Alatalo, K., et al. 2013a, MNRAS, 432, 1862 
Cappellari, M., Scott, N., Alatalo, K., et al. 2013b, MNRAS, 432, 1709

Cherinka, B., Sánchez-Gallego, J., Andrews, B., \& Brownstein, J. 2018, MNRASsdss/marvin: Marvin Beta 2.2.0, Zenodo, doi:10.5281/zenodo. 1146705

Emsellem, E., Cappellari, M., Krajnović, D., et al. 2007, MNRAS, 379, 401

Emsellem, E., Cappellari, M., Krajnović, D., et al. 2011, MNRAS, 414, 888

Fasano, G., \& Vio, R. 1991, MNRAS, 249, 629

Fogarty, L. M. R., Scott, N., Owers, M. S., et al. 2015, MNRAS, 454, 2050

Foreman-Mackey, D., Hogg, D. W., Lang, D., \& Goodman, J. 2013, PASP, 125,306

Foster, C., van de Sande, J., D’Eugenio, F., et al. 2017, MNRAS, 472, 966

Franx, M. 1988, MNRAS, 231, 285

Franx, M., Illingworth, G., \& de Zeeuw, T. 1991, ApJ, 383, 112

Graham, M. T., Cappellari, M., Li, H., et al. 2018, MNRAS, 477, 4711

Gunn, J. E., Siegmund, W. A., Mannery, E. J., et al. 2006, AJ, 131, 2332

Kimm, T., \& Yi, S. K. 2007, ApJ, 670, 1048
Krajnović, D., Emsellem, E., Cappellari, M., et al. 2011, MNRAS, 414, 2923 Krajnović, D., Emsellem, E., den Brok, M., et al. 2018, MNRAS, 477, 5327

Lambas, D. G., Maddox, S. J., \& Loveday, J. 1992, MNRAS, 258, 404

Li, H., Li, R., Mao, S., et al. 2016, MNRAS, 455, 3680

Li, H., Mao, S., Cappellari, M., et al. 2018b, MNRAS, 476, 1765

Li, H., Mao, S., Emsellem, E., et al. 2018a, MNRAS, 473, 1489

Ryden, B. 1992, ApJ, 396, 445

Sandage, A., Freeman, K. C., \& Stokes, N. R. 1970, ApJ, 160, 831

Schaller, M., Frenk, C. S., Bower, R. G., et al. 2015, MNRAS, 452, 343

Stark, A. A. 1977, ApJ, 213, 368

Statler, T. S. 1987, ApJ, 321, 113

Tsatsi, A., Lyubenova, M., van de Ven, G., et al. 2017, A\&A, 606, A62

Velliscig, M., Cacciato, M., Schaye, J., et al. 2015, MNRAS, 453, 721

Weijmans, A.-M., de Zeeuw, P. T., Emsellem, E., et al. 2014, MNRAS, 444,3340 